\title{
Prognostic Significance of Platelet-to-Lymphocyte Ratio (PLR) in Extrahepatic Metastasis of Hepatocellular Carcinoma After Curative Resection
}

\author{
Yifan Chen ${ }^{1,2, *}$ \\ Jianxing Zeng ${ }^{2, *}$ \\ Pengfei Guo ${ }^{3}$ \\ Jinhua Zeng ${ }^{1,2,4}$ \\ Jingfeng Liu (D) ${ }^{1,2,4}$
}

'Department of Hepatic Surgery, Mengchao Hepatobiliary Hospital of Fujian Medical University, Fuzhou, People's Republic of China; ${ }^{2}$ The First Affiliated Hospital of Fujian Medical University, Fuzhou, People's Republic of China; ${ }^{3}$ Southeast Big Data Institute of Hepatobiliary Health, Mengchao Hepatobiliary Hospital of Fujian Medical University, Fuzhou, People's Republic of China; ${ }^{4}$ The Liver Center of Fujian Province, Fujian Medical University, Fuzhou, People's Republic of China

*These authors contributed equally to this work
This article was published in the following Dove Press journal:

Cancer Management and Research

Background: The prognosis for patients diagnosed of hepatocellular carcinoma (HCC) who have extrahepatic metastasis after liver resection is unsatisfactory. This study aimed to find out the relationship between the inflammation-related indexes and metastasis.

Methods: One thousand three hundred and sixty-six patients diagnosed of HCC who underwent curative resection were included in this study and divided into metastasis group $(\mathrm{n}=180)$ and non-metastasis group $(\mathrm{n}=1186)$. A receiver operating characteristic (ROC) curve was constructed to estimate the optimal cut-off value for inflammation-related indexes. Independent risk factors were identified by Cox regression analysis. The metastasis rate was analyzed by the Kaplan-Meier method, then the subgroup analyses were taken.

Results: The cut-off values of NLR, PLR, LMR, N $\gamma$ LR, PNLR, and PNI were 2.65, 107.67, 5.47, 134.52, 335.03, and 51.23, respectively. Multivariate Cox analysis revealed that elevated serum AFP level $(\mathrm{P}=0.004)$, tumor size more than $5 \mathrm{~cm}(\mathrm{P}<0.001)$, multiple tumors $(\mathrm{P}=0.040)$, and higher PLR $(\mathrm{P}=0.042)$ were independent risk factors associated with extrahepatic metastasis. The Kaplan-Meier method showed that the high PLR group has a higher extrahepatic metastasis rate than the low PLR group. Meanwhile, the results of subgroup analyses were consistent with the conclusion.

Conclusion: The PLR is an independent risk factor of extrahepatic metastasis after radical hepatectomy for HCC patients. The high PLR indicates a higher rate of extrahepatic metastasis.

Keywords: hepatocellular carcinoma, liver resection, extrahepatic metastasis, inflammationrelated indexes

\section{Introduction}

Hepatocellular carcinoma is the fifth most prevalent malignancy and the thirdleading cause of cancer mortality worldwide. ${ }^{1}$ Currently, surgical resection is one of the most effective potentially curative treatments of HCC. However, the prognosis of patients with extrahepatic metastasis after curative hepatectomy is unsatisfactory, which rate is reported to be $9.8 \%-33.7 \% .^{2-5}$ Only early detection of the extrahepatic metastasis can improve survival. The pneumonectomy for pulmonary metastasis of $\mathrm{HCC}$ with well-selected patients ${ }^{6}$ or systemic therapy ${ }^{7,8}$ can prolong the survival. Therefore, it is vital to investigate the clinical characteristics and prognostic factors of these patients.
Correspondence: Jingfeng Liu Department of Hepatic Surgery, Mengchao Hepatobiliary Hospital of Fujian Medical University, Xihong Road 312, Fuzhou, 350025, People's Republic of China

$\mathrm{Tel} / \mathrm{Fax}+8659183705927$

Email drjingfeng@|26.com 
At present, there is no consensus regarding the risk factors for extrahepatic metastasis after curative intended hepatectomy for HCC. Some studies have reported that positive for hepatitis B surface antigen, ${ }^{2}$ preoperative alpha-fetoprotein (AFP) level, ${ }^{5}$ tumor size, ${ }^{4}$ and incomplete of tumor capsule ${ }^{3}$ could be the predictor of distant metastasis. However, few studies focus on the relationship between inflammation-related indexes and postoperative metastasis, although it has been widely reported to be associated with the survival and recurrence of HCC patients. ${ }^{9-15}$

Inflammation is regarded as the seventh hallmark of cancer. ${ }^{16}$ It can create a tissue microenvironment characterized by tissue rearrangement, immunosuppression, and abundant growth factors that allow the tumor to initiate, progress, and metastasize through altering the local control of tissue homeostasis, cell proliferation, and genetic stability. ${ }^{17}$ Some inflammation-related indexes have been reported as independent risk factors for prognosis in HCC patients after liver resection, such as the neutrophil-to-lymphocyte ratio (NLR), ${ }^{18}$ platelet-to-lymphocyte ratio (PLR), ${ }^{10}$ lymphocyte-tomonocyte ratio (LMR), ${ }^{12}$ neutrophil times $\gamma$-glutamyl transpeptidase-to-lymphocyte ratio $(\mathrm{N} \gamma \mathrm{LR}),{ }^{14}$ platelet times neutrophil-to-lymphocyte ratio (PNLR), ${ }^{15}$ prognostic nutritional index (PNI). ${ }^{9}$ However, the role of inflammation-related indexes in extrahepatic metastasis after curative hepatectomy has not been well illustrated.

In this study, we conducted a large patient cohort to systematically explore the impact of inflammation-related indexes on patients with extrahepatic metastasis after curative intended hepatectomy for HCC.

\section{Patients and Methods}

\section{Patients}

Between January 2011 and December 2013, data of patients with HCC underwent primary hepatectomy extracted from primary liver cancer big data were retrospectively analyzed.

The inclusion criteria were as follows: (1) HCC was confirmed by histopathological examination; (2) curative resection with tumor-negative resection margins (R0 resection); (3) no evidence of extrahepatic metastasis; (4) no macrovascular invasion; and (5) without infectionrelated or hematological system disease. Patients who received preoperative anti-tumor treatments underwent palliative tumor resection, had a history of or accompany with other cancer, had incomplete data, lost to follow-up within 60 days after operation were excluded from the analysis.

The study was conducted according to the principles of the Declaration of Helsinki and approved by the Institutional Ethics Committee of Mengchao Hepatobiliary Hospital of Fujian Medical University (NO.: 2020_116_01). All patients were informed consent for their data to be used for research purposes.

\section{Clinicopathologic Factors}

Clinical data included patients' age, sex, Barcelona Clinic Liver Cancer (BCLC) stage, and the 8th American Joint Committee on Cancer (AJCC) stage were collected and assessed within seven days before operation. Routine peripheral blood samples of all patients were obtained within two weeks before surgery including white blood cell count (WBC), red blood cell count (RBC), neutrophil count, monocyte count, lymphocyte count, platelet count (PLT), hemoglobin (HB), albumin (ALB), total bilirubin (TBil), gamma-glutamyl transpeptidase $(\gamma-\mathrm{GT})$, hepatitis B surface antigen ( $\mathrm{HBsAg}$ ), and hepatitis B virus DNA (HBV-DNA). The pathological data confirmed by two experienced pathologists were also included: surgical margin status, tumor number, tumor size, microvascular invasion (MVI) status, differentiation grade, tumor capsule, satellite, and cirrhosis. Then the inflammation-related indexes were calculated by the formula as follows: NLR as the division of neutrophil count by lymphocyte count, PLR as the division of platelet count by lymphocyte count, LMR as the division of lymphocyte count by monocyte count, $\mathrm{N} \gamma \mathrm{LR}$ as the neutrophil count times $\gamma$-GT count then divided by the lymphocyte count, PNLR as the platelet count times neutrophil count then divided by the lymphocyte count, PNI as (10 times serum albumin $[\mathrm{g} / \mathrm{dL}])$ plus (0.005 times lymphocyte count $[/ \mathrm{mm} 3]){ }^{9}$

\section{Follow-Up}

Patients were followed up once every three months for the first two years after discharge from the hospital and every three to six months after that. During the followup program, not only the blood samples for the 
complete blood cell count, liver function test, and tumor markers, but also the imaging examination such as abdominal ultrasonography, contrast-enhanced abdominal computed tomography (CT), and abdominal magnetic resonance imaging (MRI) are required. According to the decision made by experienced clinicians depending on the serum AFP rising or clinical symptoms, the metastasis-related monitored examination was taken, such as chest computed tomography, brain computed tomography, and radionuclide bone imaging. The follow-up on 31 October 2018 was censored.

\section{Statistical Analysis}

Categorical variables were using Chi-square or Fisher exact tests for comparative analysis and presented as a frequency in our retrospective study. Continuous variables were adopted Student's $t$-test or Mann-Whitney $U$-test for comparison and demonstrate with a mean (standard deviation, SD) or median (interquartile range, IQR). A ROC curve was constructed to estimate the optimal cut-off value for NLR, PLR, LMR, N $\gamma L R$, and PNI calculated by the formula above. A Cox proportional hazards model was used for univariate and multivariate analysis to detect the independent factors associated with metastasis. Factors with $\mathrm{P}<0.05$ in the aforementioned univariate Cox model comparisons were included in a multivariate analysis. Data from these models were depicted with hazard ratios (HRs) and $95 \%$ confidence intervals (CIs). The metastasis rate was analyzed by the Kaplan-Meier method, and the Log rank test was adopted to compare the difference between groups. All the p-values were two-tailed and less than 0.05 was considered statistically significant. Our statistical analyses were performed with $\mathrm{R}$ version 4.0.2 (http://www.r-project.org/).

\section{Results}

\section{Baseline Clinicopathologic Characteristics}

A total of 1851 patients who underwent radical resection who met our inclusion criteria were enrolled in our study from the primary liver cancer big data, during January 2011 and December 2013. Four hundred and ninety-five patients were excluded because of preoperative anticancer treatment $(\mathrm{n}=154)$, a history of other malignancies $(\mathrm{n}=15)$, and incomplete clinical or follow-up data $(n=316)$. Finally, the study cohort consisted of 1366 patients, which comprises 180 patients with metastasis after hepatectomy and 1186 without. The flow chart of this patient selection is shown in Figure 1 .

The median value of NLR, PLR, LMR, N $\gamma L R$, PNLR, and PNI were 1.89, 96.55, 5.22, 108.47, 277.93, and 49.85. We conducted the ROC curve analysis to decide the optimal cut-off values for the inflammation-related indexes distinguished from the high and low groups. As demonstrated in Table 1, the cut-off values of NLR, PLR, LMR, N $\gamma L R$, PNLR, and PNI were 2.65, 107.67, 5.47, 134.52, 335.03, and 51.23 , respectively.

The comparison of the two groups is summarized in Table 2. There was no significant difference between the two groups of age and gender. Considering the peripheral blood sample, the metastasis group had higher WBC $\quad(\mathrm{P}=0.036), \quad \mathrm{PLT} \quad(\mathrm{P}=0.008), \quad \mathrm{GGT}$ $(\mathrm{P}=0.005)$, and AFP $(\mathrm{P}=0.001)$ than the non-metastasis group. More blood loss $(\mathrm{P}=0.005)$ and blood transfusion $(\mathrm{P}=0.003)$ during the surgery were more in the metastasis group than the non-metastasis group. As the pathological related tumor factors, the metastasis groups had larger tumor diameter $(\mathrm{P}<0.001)$, more tumor number $(\mathrm{P}=0.005)$, higher rates of Edmondson grade III-IV $(\mathrm{P}=0.020)$ and liver cirrhosis $(\mathrm{P}=0.047)$, more advanced BCLC stages $(p=0.002)$ and more advanced AJCC TNM stages $(p<0.001)$ than the nonmetastasis group. All the inflammation-related indexes demonstrated the significant difference between the two groups, including NLR $(\mathrm{P}<0.001)$, PLR $(\mathrm{P}<0.001)$, LMR $(\mathrm{P}=0.002), \mathrm{N} \gamma \mathrm{LR}(\mathrm{P}<0.001)$, PNLR $(\mathrm{P}<0.001)$, PNI $(\mathrm{P}=0.010)$.

\section{Univariate and Multivariate Cox Analysis for Extrahepatic Metastasis in HCC Patients After Radical Resection}

The univariate and multivariate Cox analysis for determining the risk factors associated with extrahepatic metastasis in HCC patients after radical resection were shown in Table 3. Univariate analysis identified elevated serum AFP level $(\mathrm{P}<0.001)$, bleeding loss more than $800 \mathrm{~mL}$ $(\mathrm{P}<0.001)$ and transfusion during operation $(\mathrm{P}<0.001)$, tumor size more than $5 \mathrm{~cm}(\mathrm{P}<0.001)$, multiple tumors $(\mathrm{P}<0.001)$, presence of MVI $(\mathrm{P}=0.006)$, higher rates of 
HCC patients who underwent radical resection between 2011 and

2013 was extracted from primary liver cancer big data $(n=1851)$

Excluded patients $(n=485)$ :

Preoperative anticancer treatment $(n=154)$

History of other malignancies $(n=15)$

Incomplete clinical and follow-up data $(n=316)$

$\begin{gathered}\text { Patients with } \\ \text { metastasis } \\ (n=180)\end{gathered}$
(n)
metastasis
$(n=1186)$

Figure I The flow chart of selected patients.

Abbreviation: HCC, hepatocellular carcinoma.

Edmondson grade III-IV ( $\mathrm{P}=0.006)$, presence of satellite nodules $(\mathrm{P}=0.017)$, higher NLR $(\mathrm{P}<0.001)$, higher PLR $(\mathrm{P}<0.001)$, lower LMR $(\mathrm{P}<0.001)$, higher $\mathrm{N} \gamma \mathrm{LR}$ $(\mathrm{P}<0.001)$, higher PNLR $(\mathrm{P}<0.001)$, and lower PNI $(\mathrm{P}=0.003)$ were associated with extrahepatic metastasis.
In addition, the multivariate analysis revealed that elevated serum AFP level $(\mathrm{P}=0.004)$, tumor size more than $5 \mathrm{~cm}$ $(\mathrm{P}<0.001)$, multiple tumors $(\mathrm{P}=0.040)$, and higher $\mathrm{PLR}$ $(\mathrm{P}=0.042)$ were independent risk factors associated with extrahepatic metastasis.

Table I The Optimal Cut-Off Value for NLR, PLR, LMR, PNI, and N $\gamma$ LR Calculated by the ROC Curve

\begin{tabular}{|l|c|c|c|c|c|c|}
\hline Variables & Cut-Off Value & AUC & $\mathbf{9 5 \%} \mathbf{~ C l}$ & Sensitivity & Specificity & p-value \\
\hline NLR & 2.65 & 0.573 & $0.527-0.619$ & 0.333 & 0.789 & 0.002 \\
PLR & 107.67 & 0.592 & $0.547-0.637$ & 0.572 & 0.621 & $<0.001$ \\
LMR & 5.47 & 0.554 & $0.509-0.599$ & 0.480 & 0.650 & 0.019 \\
PNI & 51.23 & 0.552 & $0.509-0.595$ & 0.398 & 0.706 & 0.024 \\
N $\gamma$ LR & 134.52 & 0.620 & $0.577-0.663$ & 0.578 & 0.619 & $<0.001$ \\
PNLR & 335.03 & 0.594 & $0.548-0.639$ & 0.522 & 0.636 & $<0.001$ \\
\hline
\end{tabular}

Abbreviations: AUC, area under the ROC curve; Cl, confidence interval; NLR, neutrophil-to-lymphocyte ratio; PLR, platelet-to-lymphocyte ratio; LMR, lymphocyte-tomonocyte ratio; PNI, prognostic nutritional index; $\mathrm{N} \gamma \mathrm{LR}$, (neutrophil times $\gamma$-GT)-to-lymphocyte ratio. 
Table 2 Comparison of Clinicopathological Characteristics Between Metastasis and Non-Metastasis

\begin{tabular}{|c|c|c|c|}
\hline Variables & $\begin{array}{l}\text { Non-Metastasis } \\
\text { Group }(n=\mid \text { | 86) }\end{array}$ & $\begin{array}{c}\text { Metastasis } \\
\text { Group }(n=180)\end{array}$ & $\begin{array}{c}\text { p- } \\
\text { value }\end{array}$ \\
\hline $\begin{array}{l}\text { Age, years, } \\
\text { Median [IQR] }\end{array}$ & $52.0[45.0,60.0]$ & $50.0[43.0,60.0]$ & 0.334 \\
\hline $\begin{array}{c}\text { Gender } \\
\text { Female } \\
\text { Male }\end{array}$ & $\begin{array}{c}170(14.3 \%) \\
1016(85.7 \%)\end{array}$ & $\begin{array}{l}22(\mid 2.2 \%) \\
\text { I58 (87.8\%) }\end{array}$ & 0.519 \\
\hline $\begin{array}{l}\text { BCLC } \\
\text { Stage } 0 \\
\text { Stage A } \\
\text { Stage B }\end{array}$ & $\begin{array}{c}60(5.1 \%) \\
955(80.5 \%) \\
171(14.4 \%)\end{array}$ & $\begin{array}{c}4(2.2 \%) \\
133(73.9 \%) \\
43(23.9 \%)\end{array}$ & 0.002 \\
\hline $\begin{array}{l}\text { TNM stage } \\
\text { (8th) } \\
\text { IA } \\
\text { IB } \\
\text { II } \\
\text { IIIA }\end{array}$ & $\begin{array}{c}68(5.7 \%) \\
713(60.1 \%) \\
305(25.7 \%) \\
100(8.4 \%)\end{array}$ & $\begin{array}{c}4(2.2 \%) \\
94(52.2 \%) \\
51(28.3 \%) \\
31(17.2 \%)\end{array}$ & $<0.001$ \\
\hline $\begin{array}{l}\text { HBsAg } \\
\text { Negative } \\
\text { Positive }\end{array}$ & $\begin{array}{c}116(9.8 \%) \\
1070(90.2 \%)\end{array}$ & $\begin{array}{l}23(12.8 \%) \\
157(87.2 \%)\end{array}$ & 0.268 \\
\hline $\begin{array}{l}\text { HBV-DNA } \\
\quad<10001 \mathrm{I} / \mathrm{mL} \\
\geq 1000 \mathrm{IU} / \mathrm{mL}\end{array}$ & $\begin{array}{l}663(55.9 \%) \\
523(44.1 \%)\end{array}$ & $\begin{array}{l}103(57.2 \%) \\
77(42.8 \%)\end{array}$ & 0.801 \\
\hline $\begin{array}{l}\text { AFP, } \mathbf{n g} / \mathbf{m L} \text {, } \\
\text { Median [IQR] }\end{array}$ & $42.6[5.40,692]$ & $87.1[8.33,1210]$ & 0.001 \\
\hline $\begin{array}{l}\text { WBC, } 10^{9} / \mathrm{L} \\
\text { Mean (SD) }\end{array}$ & $5.25(1.54)$ & 5.55 (I.79) & 0.036 \\
\hline $\begin{array}{l}\text { RBC, } 10^{12} / \mathrm{L}, \\
\text { Mean (SD) }\end{array}$ & $4.61(0.522)$ & $4.69(0.620)$ & 0.127 \\
\hline $\begin{array}{l}\text { HB, g/L, } \\
\text { Mean (SD) }\end{array}$ & $142(16.0)$ & 142 (I5.9) & 0.912 \\
\hline $\begin{array}{l}\text { PLT, } 10^{9} / \mathrm{L}, \\
\text { Mean (SD) }\end{array}$ & $160(68.5)$ & I 75 (70.7) & 0.008 \\
\hline $\begin{array}{l}\text { ALB, g/L, } \\
\text { Mean (SD) }\end{array}$ & 42.1 (3.70) & 41.6 (3.39) & 0.069 \\
\hline $\begin{array}{l}\text { TBIL, } \boldsymbol{\mu m o l} / \mathbf{L} \text {, } \\
\text { Median [IQR] }\end{array}$ & $13.2[10.5,16.6]$ & $\mid 2.6[10.2, \mid 6.5]$ & 0.420 \\
\hline $\begin{array}{l}\text { GGT, U/L, } \\
\text { Median [IQR] }\end{array}$ & $54.0[32.0,97.0]$ & $76.0[42.0,124]$ & 0.005 \\
\hline $\begin{array}{l}\text { Surgical } \\
\text { margin width } \\
\quad<I \mathrm{~cm} \\
\geq \mathrm{Icm}\end{array}$ & $\begin{array}{l}962(81.1 \%) \\
224(18.9 \%)\end{array}$ & $\begin{array}{c}150(83.3 \%) \\
30(16.7 \%)\end{array}$ & 0.541 \\
\hline
\end{tabular}

(Continued)
Table 2 (Continued).

\begin{tabular}{|c|c|c|c|}
\hline Variables & $\begin{array}{l}\text { Non-Metastasis } \\
\text { Group }(n=\mid \text { | 86) }\end{array}$ & $\begin{array}{c}\text { Metastasis } \\
\text { Group }(n=\mid 80)\end{array}$ & $\begin{array}{c}\text { p- } \\
\text { value }\end{array}$ \\
\hline \multicolumn{4}{|l|}{$\begin{array}{l}\text { Blood } \\
\text { transfusion }\end{array}$} \\
\hline NO & 1106 (93.3\%) & I 56 (86.7\%) & 0.003 \\
\hline YES & 80 (6.7\%) & 24 (I3.3\%) & \\
\hline \multicolumn{4}{|l|}{$\begin{array}{l}\text { Operative } \\
\text { bleeding loss }\end{array}$} \\
\hline$<800 \mathrm{~mL}$ & 1113 (93.8\%) & I 58 (87.8\%) & 0.005 \\
\hline$\geq 800 \mathrm{~mL}$ & $73(6.2 \%)$ & $22(12.2 \%)$ & \\
\hline \multicolumn{4}{|l|}{$\begin{array}{l}\text { Tumor } \\
\text { diameter }\end{array}$} \\
\hline$<5 \mathrm{~cm}$ & 662 (55.8\%) & 58 (32.2\%) & $<0.001$ \\
\hline$\geq 5 \mathrm{~cm}$ & 524 (44.2\%) & I 22 (67.8\%) & \\
\hline \multicolumn{4}{|l|}{$\begin{array}{l}\text { Tumor } \\
\text { number }\end{array}$} \\
\hline Single & 984 (83.0\%) & I33 (73.9\%) & 0.005 \\
\hline Multiple & 202 (I7.0\%) & 47 (26.1\%) & \\
\hline \multicolumn{4}{|l|}{$\begin{array}{l}\text { Microvascular } \\
\text { invasion }\end{array}$} \\
\hline NO & 888 (74.9\%) & I 24 (68.9\%) & 0.106 \\
\hline YES & $298(25.1 \%)$ & $56(31.1 \%)$ & \\
\hline \multicolumn{4}{|l|}{$\begin{array}{l}\text { Edmondson } \\
\text { grade }\end{array}$} \\
\hline I-II & 155 (13.1\%) & $12(6.7 \%)$ & 0.020 \\
\hline III-IV & $103 \mid$ (86.9\%) & 168 (93.3\%) & \\
\hline \multicolumn{4}{|l|}{$\begin{array}{l}\text { Tumor } \\
\text { capsule }\end{array}$} \\
\hline NO & 204 (I7.2\%) & 37 (20.6\%) & 0.320 \\
\hline YES & $982(82.8 \%)$ & I 43 (79.4\%) & \\
\hline \multicolumn{4}{|l|}{$\begin{array}{l}\text { Satellite } \\
\text { nodules }\end{array}$} \\
\hline NO & 778 (65.6\%) & 107 (59.4\%) & 0.127 \\
\hline YES & 408 (34.4\%) & 73 (40.6\%) & \\
\hline \multicolumn{4}{|l|}{ Liver cirrhosis } \\
\hline NO & 388 (32.7\%) & 73 (40.6\%) & 0.047 \\
\hline YES & 798 (67.3\%) & 107 (59.4\%) & \\
\hline \multicolumn{4}{|l|}{ NLR } \\
\hline$<2.65$ & 936 (78.9\%) & I 20 (66.7\%) & $<0.001$ \\
\hline$\geq 2.65$ & $250(21.1 \%)$ & $60(33.3 \%)$ & \\
\hline \multicolumn{4}{|l|}{ PLR } \\
\hline$<107.67$ & 736 (62.1\%) & 77 (42.8\%) & $<0.001$ \\
\hline$\geq 107.67$ & 450 (37.9\%) & 103 (57.2\%) & \\
\hline \multicolumn{4}{|l|}{ LMR } \\
\hline$<5.47$ & 617 (52.0\%) & II (65.0\%) & 0.002 \\
\hline$\geq 5.47$ & 569 (48.0\%) & 63 (35.0\%) & \\
\hline
\end{tabular}

(Continued) 
Table 2 (Continued).

\begin{tabular}{|l|c|c|c|}
\hline Variables & $\begin{array}{c}\text { Non-Metastasis } \\
\text { Group (n= I I 86) }\end{array}$ & $\begin{array}{c}\text { Metastasis } \\
\text { Group (n= I 80) }\end{array}$ & $\begin{array}{c}\text { p- } \\
\text { value }\end{array}$ \\
\hline $\begin{array}{c}\text { N } \gamma \text { LR } \\
<134.52\end{array}$ & $734(61.9 \%)$ & $76(42.2 \%)$ & $<0.001$ \\
$\geq 134.52$ & $452(38.1 \%)$ & $104(57.8 \%)$ & \\
\hline PNLR & & & \\
$<335.03$ & $754(63.6 \%)$ & $86(47.8 \%)$ & $<0.001$ \\
$\geq 335.03$ & $432(36.4 \%)$ & $94(52.2 \%)$ & \\
\hline PNI & $714(60.2 \%)$ & $127(70.6 \%)$ & 0.010 \\
$<51.23$ & $472(39.8 \%)$ & $53(29.4 \%)$ & \\
\hline 51.23 & & & \\
\hline
\end{tabular}

Abbreviations: BCLC, Barcelona Clinic Liver Cancer; TNM, tumor-node-metastases; $\mathrm{HBsAg}$, hepatitis $B$ surface antigen; HBV-DNA, hepatitis $B$ virus deoxyribonucleic acid; AFP, alpha-fetoprotein; WBC, white blood cell; RBC, red blood cell; HB, hemoglobin; PLT, platelet count; ALB, albumin; TBIL, total bilirubin; GGT, gamma-glutamyltransferase; SD, standard deviation; IQR, interquartile range; NLR, neutrophil-to-lymphocyte ratio; PLR, platelet-to-lymphocyte ratio; LMR, lymphocyte-to-monocyte ratio; PNI, prognostic nutritional index; $\mathrm{N} \gamma \mathrm{LR}$, (neutrophil times $\gamma$-GT)-to-lymphocyte ratio.

\section{Comparison of Metastasis Between Low and High PLR Group in HCC Patients After Radical Resection}

The metastasis rate in HCC patients after radical liver resection between high and low PLR group is shown in Figure 2. The cumulated metastasis rates at 1, 3, 5 years were $4.80 \%, 7.87 \%$, and $8.98 \%$, respectively, in the low PLR group and $13.38 \%, 16.64 \%$, and $18.44 \%$, respectively, in the high PLR group $(\mathrm{p}<0.0001)$.

To validate in which optimal group that the different grade PLR could distinguish metastasis for HCC patients after surgery, subgroup analyses were conducted based on staging system, different serum AFP level, liver cirrhosis status, and hepatitis B infection status.

Based on the BCLC staging system, 64 (4.7\%), $1088(79.6 \%)$, and $214(15.7 \%)$ patients were assigned to stage $0 / \mathrm{A} / \mathrm{B}$ groups. As for stage $0 / \mathrm{A}$, patients in the low PLR group had a lower extrahepatic metastasis rate than the high PLR group $(\mathrm{P}<0.001$, Figure $3 \mathrm{~A})$. We detected a similar outcome in stage $\mathrm{B}(\mathrm{P}<0.001$, Figure 3B).

Considering the different preoperative serum AFP level, we divided it into three groups less than $20 \mathrm{ng} /$ $\mathrm{mL}, 20$ to $400 \mathrm{ng} / \mathrm{mL}$, and no less than $400 \mathrm{ng} / \mathrm{mL}$, which including 564 (41.3\%), 387 (28.3\%), 415
(30.3\%) patients, respectively. Kaplan-Meier analysis showed that the PLR could well stratify the metastasis rates among three risk groups (Figure 4).

As for cirrhosis status, there were 905 (66.3\%) patients with pathological liver cirrhosis confirmed and 461 (33.7\%) patients without. Both the cirrhosis group $(\mathrm{P}<0.001)$ and the non-cirrhosis group $(\mathrm{P}=0.001)$ have a consistent result of the high PLR cohort was more likely to have metastasis than the low PLR cohort (Figure 5).

Besides, there were $1227 \mathrm{HBsAg}$ positive and 139 HBsAg negative patients in our cohort. Both HBsAg positive group $(\mathrm{P}<0.001)$ and $\mathrm{HBsAg}$ negative group $(\mathrm{P}=0.002)$ showed a higher metastasis rate in the high PLR group than in the low PLR group (Figure 6).

\section{Discussions}

Nowadays, hepatectomy remains the most effective and common treatment strategy for HCC. However, the rate of extrahepatic metastasis after curative resection for HCC patients is becoming higher due to the progress of standardized follow-up plan and imaging examination techniques. Once extrahepatic metastasis is developed, the prognosis is poorer than those who have not recurrence or has intrahepatic recurrence only. ${ }^{19}$ At present, the factors associated with extrahepatic metastasis were not consistent. Identification of the specific factors for predicting extrahepatic metastasis is necessary.

Based on our large cohort, the multivariate analysis showed that elevated serum AFP level, high PLR, multiple tumor number, and tumor diameter no less than $5 \mathrm{~cm}$ were independent risk factors. As the commonly used staging systems, the Barcelona Clinic Liver Cancer (BCLC), ${ }^{20}$ and the 8th American Joint Committee on Cancer (AJCC) ${ }^{21}$ have been proposed using some factors, including tumor size and tumor number for risk stratification. These two factors are also associated with extrahepatic metastasis according to our results, which was consistent with some other researches, ${ }^{4,22}$ AFP has been reported to not only be an important role in oncogenesis, growth, and metastasis in liver cancer but also prevents apoptosis and escaping of HCC from immune surveillance. ${ }^{23}$ Several studies report that patients with elevated serum AFP level have a poor prognosis, ${ }^{5,22}$ In our research, the elevated AFP 
Table 3 Univariate and Multivariate Cox Regression for Extrahepatic Metastasis in HCC Patients After Radical Resection

\begin{tabular}{|c|c|c|c|c|c|c|}
\hline \multirow[t]{2}{*}{ Variables } & \multicolumn{3}{|c|}{ Univariate } & \multicolumn{3}{|c|}{ Multivariate } \\
\hline & HR & $95 \% \mathrm{Cl}$ & p-value & HR & $95 \% \mathrm{Cl}$ & p-value \\
\hline Age: $\geq 60$ year vs $<60$ year & 1.012 & $0.727-1.408$ & 0.946 & & & \\
\hline Gender: male vs female & 1.208 & $0.773-1.887$ & 0.407 & & & \\
\hline HBsAg: positive vs negative & $0.76 \mathrm{I}$ & $0.491-1.178$ & 0.221 & & & \\
\hline HBV-DNA: $\geq 10001 \mathrm{U} / \mathrm{mL}$ vs $<10001 \mathrm{I} / \mathrm{mL}$ & 1.009 & $0.75 I-1.356$ & 0.953 & & & \\
\hline AFP, $n g / m L$ & 1.001 & $1.000-1.001$ & $<0.001$ & 1.001 & $1.000-1.001$ & 0.004 \\
\hline $\mathrm{RBC}, 10^{12} / \mathrm{L}$ & 1.265 & $0.962-1.664$ & 0.093 & & & \\
\hline $\mathrm{HB}, \mathrm{g} / \mathrm{L}$ & 1.000 & $0.991-1.009$ & 0.991 & & & \\
\hline TBIL, $\mu \mathrm{mol} / \mathrm{L}$ & 0.989 & $0.96 I-1.018$ & 0.451 & & & \\
\hline Surgical margin width: $\geq I \mathrm{~cm}$ vs $<I \mathrm{~cm}$ & 0.817 & $0.552-1.209$ & 0.311 & & & \\
\hline Blood transfusion: yes vs no & 2.269 & $1.475-3.488$ & $<0.001$ & 1.157 & $0.577-2.320$ & 0.681 \\
\hline Operative bleeding loss: $\geq 800 \mathrm{~mL}$ vs $<800 \mathrm{~mL}$ & 2.397 & $1.534-3.748$ & $<0.001$ & 1.220 & $0.591-2.518$ & 0.591 \\
\hline Tumor diameter: $\geq 5 \mathrm{~cm}$ vs $<5 \mathrm{~cm}$ & 2.843 & $2.078-3.888$ & $<0.001$ & 1.926 & $1.355-2.737$ & $<0.001$ \\
\hline Tumor number: multiple vs single & 1.882 & $1.349-2.626$ & $<0.001$ & 1.611 & $1.021-2.542$ & 0.040 \\
\hline Microvascular invasion: yes vs no & 1.554 & $1.133-2.132$ & 0.006 & 1.308 & $0.930-|.84|$ & 0.123 \\
\hline Edmondson grade: III-IV vs I-II & 2.258 & $1.257-4.057$ & 0.006 & 1.351 & $0.735-2.482$ & 0.332 \\
\hline Tumor capsule: yes vs no & 0.736 & $0.513-1.057$ & 0.097 & & & \\
\hline Satellite nodules: yes vs no & 1.435 & $1.065-1.933$ & 0.017 & 0.994 & $0.650-1.520$ & 0.978 \\
\hline Liver cirrhosis: yes vs no & 0.747 & $0.555-1.006$ & 0.054 & & & \\
\hline NLR: $\geq 2.65$ vs $<2.65$ & 1.881 & I.380-2.565 & $<0.001$ & 0.990 & $0.665-1.473$ & 0.959 \\
\hline PLR: $\geq 107.67$ vs $<107.67$ & 2.190 & $1.630-2.943$ & $<0.001$ & 1.488 & $1.015-2.184$ & 0.042 \\
\hline LMR: $\geq 5.47$ vs $<5.47$ & 0.562 & $0.4 \mid 4-0.764$ & $<0.001$ & 0.831 & $0.588-1.174$ & 0.294 \\
\hline N $\gamma$ LR: $\geq 134.52$ vs $<134.52$ & 2.351 & $1.748-3.16 \mid$ & $<0.001$ & 1.397 & $0.987-1.978$ & 0.059 \\
\hline PNLR: $\geq 335.03$ vs $<335.03$ & 1.875 & $1.400-2.513$ & $<0.001$ & 0.894 & $0.591-1.354$ & 0.598 \\
\hline PNI: $\geq 51.23$ vs $<5 I .23$ & 0.617 & $0.448-0.851$ & 0.003 & 0.784 & $0.556-1.105$ & 0.165 \\
\hline
\end{tabular}

Abbreviations: $\mathrm{HR}$, hazard ratio; $\mathrm{Cl}$, confidence interval; $\mathrm{HBsAg}$, hepatitis B surface antigen; HBV-DNA, hepatitis B Virus deoxyribonucleic acid; AFP, alpha-fetoprotein; $\mathrm{RBC}$, red blood cell; HB, hemoglobin; TBIL, total bilirubin; NLR, neutrophil-to-lymphocyte ratio; PLR, platelet-to-lymphocyte ratio; LMR, lymphocyte-to-monocyte ratio; $\mathrm{PNI}$, prognostic nutritional index; $\mathrm{N} \gamma \mathrm{LR}$, (neutrophil times $\gamma$-GT)-to-lymphocyte ratio.

level is an independent risk factor for extrahepatic metastasis, which contributes to the poor prognosis.

Many studies have found that inflammation plays a crucial role in the pathogenesis and progression of HCC. ${ }^{9,10,12-18}$ However, few studies focus on the association between inflammation-related indexes and extrahepatic metastasis after radical hepatectomy for HCC. In this study, we adopted several common inflammation-related indexes, using a large cohort to find out only the PLR is an independent risk factor for extrahepatic metastasis. The PLR is a commonly used inflammationrelated index, which is a combination of peripheral platelet 


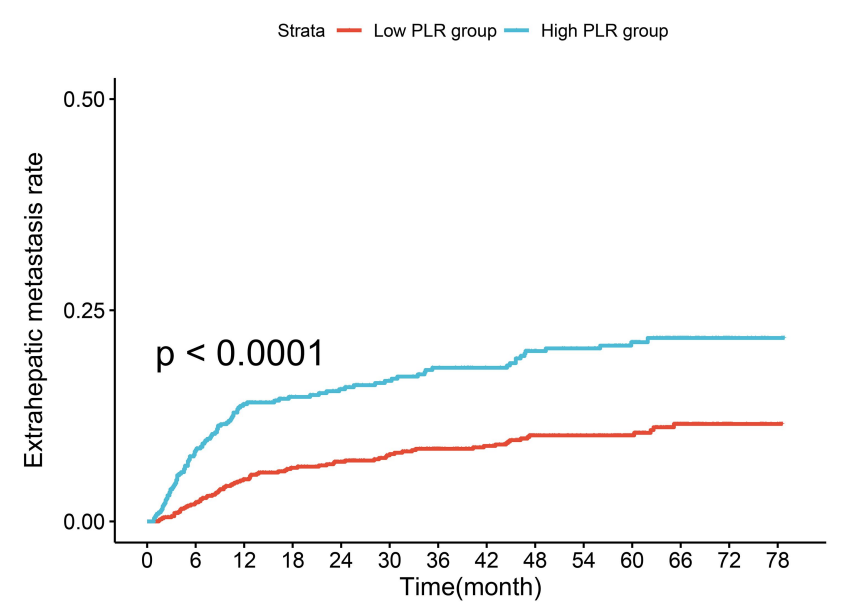

Number at risk

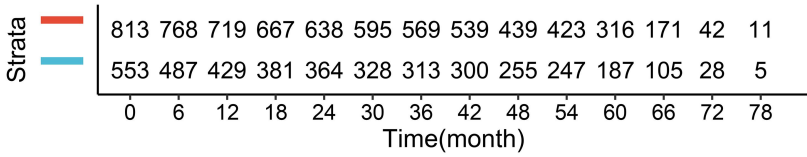

Figure 2 Comparison of metastasis rate between high and low PLR groups. Abbreviation: PLR, platelet-to-lymphocyte ratio.

and lymphocyte count. Bihari et al suggested that platelets could be associated with the development, growth, invasion, and metastasis of HCC and could be regarded as a prognostic marker in $\mathrm{HCC}^{24}$ Chew et al considered that lymphocytes could affect patient survival through mechanisms leading to the inhibition of tumor survival or proliferation. ${ }^{25}$ Thus, the high PLR, which means higher platelet counts and lower lymphocyte counts suggest higher rates of extrahepatic metastasis.

Furthermore, whether the PLR could be suitable for distinguishing all the metastasis rates of HCC patients after radical hepatectomy is of concern. We put forward the subgroup analysis of the BCLC staging system, different serum AFP levels, liver cirrhosis status, and hepatitis B infection status. As we know, the BCLC staging system adopted tumor size and tumor number to distinguish stages $0, \mathrm{~A}$, and $\mathrm{B}$, which including all the stages of our cohort. However, the different PLR group could also predict statistically significant different prognosis among the three stages. AFP is the most commonly used tumor marker for HCC. We divided it into a normal level group $(<20$ $\mathrm{ng} / \mathrm{mL}$ ), abnormal level group (20-400 $\mathrm{ng} / \mathrm{mL}$ ), and higher-level group ( $>400 \mathrm{ng} / \mathrm{mL})$. Using the KaplanMeier method, the high PLR group showed higher rates of metastasis than the low PLR group among three different AFP level groups. Even in the normal AFP level group, the PLR demonstrated that it is capable of distinguishing the metastasis rate. Liver cirrhosis has been reported to be associated with systemic
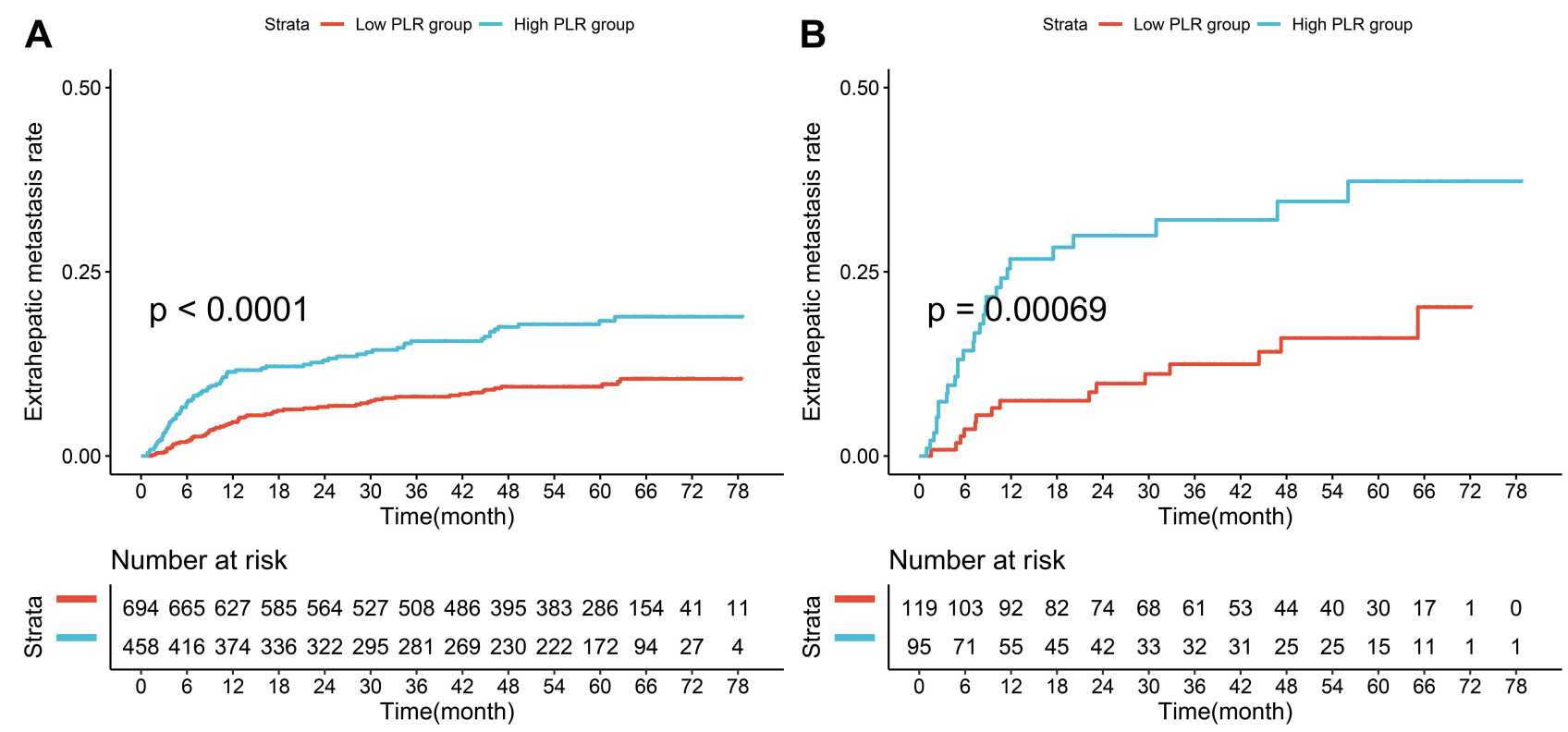

Figure 3 Subgroup analysis of metastasis rate between high and low PLR groups. (A) Metastasis rate in the BCLC stage 0/A patients; (B) metastasis rate in the BCLC stage B patients.

Abbreviations: PLR, platelet-to-lymphocyte ratio; BCLC, Barcelona Clinic Liver Cancer. 

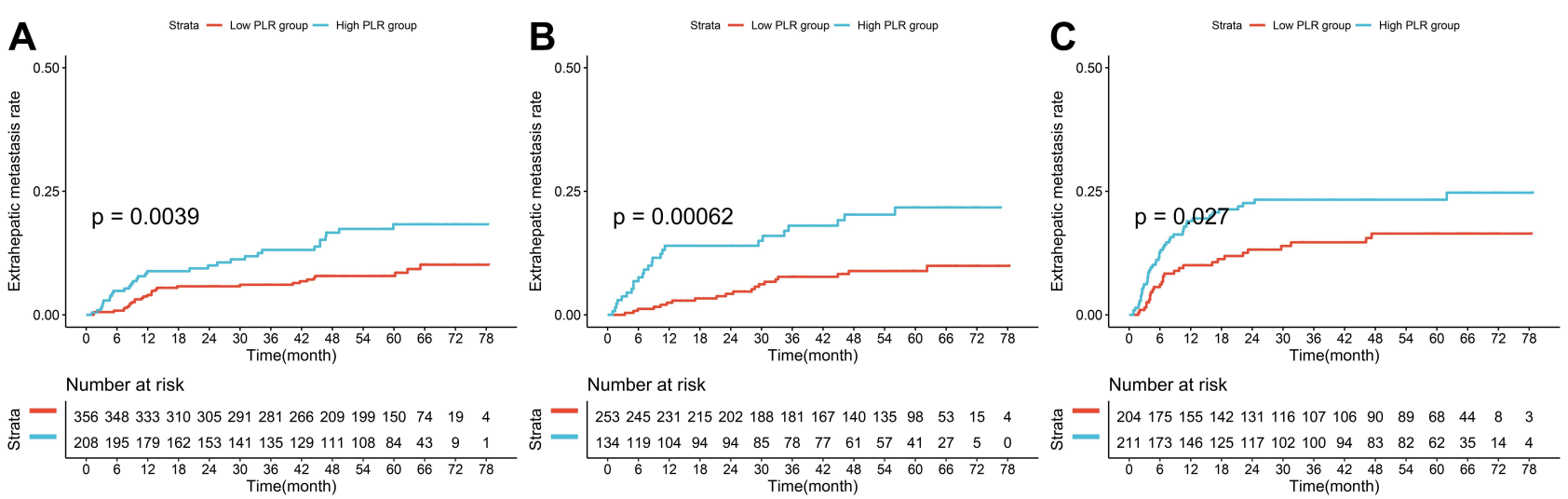

Number at risk

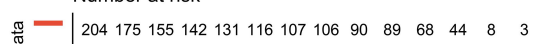

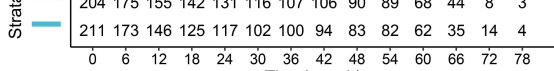

Figure 4 Subgroup analysis of metastasis rate between high and low PLR groups. (A) Metastasis rate in the normal AFP level group (<20ng/mL); (B) metastasis rate in the abnormal AFP level group ( $\geq 20 \mathrm{ng} / \mathrm{mL}$ and $<400 \mathrm{ng} / \mathrm{mL})$; (C) metastasis rate in the higher AFP level group $(\geq 400 \mathrm{ng} / \mathrm{mL})$.

Abbreviations: PLR, platelet-to-lymphocyte ratio; AFP, alpha-fetoprotein.

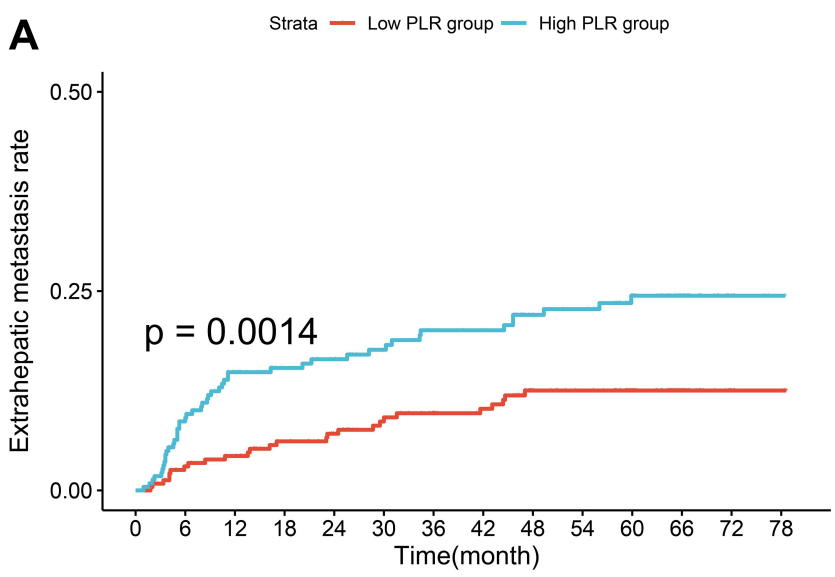

Number at risk

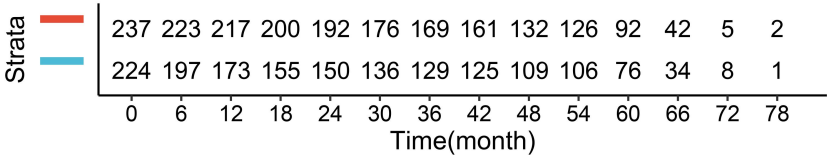

B Strata - Low PLR group — High PLR group

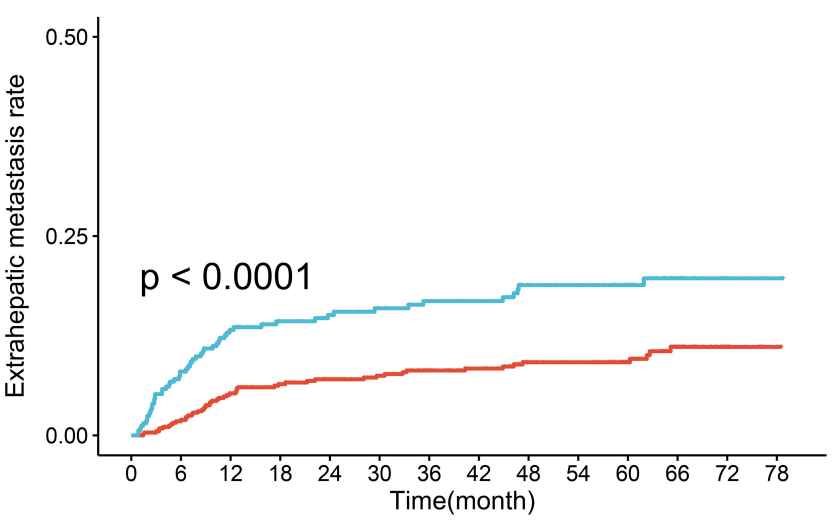

Number at risk

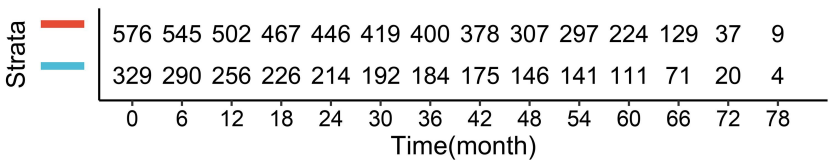

Figure 5 Subgroup analysis of metastasis rate between high and low PLR groups. (A) Metastasis rate in the non-cirrhosis group; (B) metastasis rate in the cirrhosis group. Abbreviation: PLR, platelet-to-lymphocyte ratio.

inflammation. ${ }^{26,27}$ Although liver cirrhosis may influence inflammation-related indexes, subgroup analysis is consistent with that the high PLR group has a higher rate of metastasis regardless of cirrhosis status.

There are several limitations to our study. First, the cut-off values of the inflammation-related indexes have not a uniform standard. To solve this problem, we conducted the ROC curve to decide the value for our research, which could be different while using for other cohorts. Second, nearly $90 \%$ of patients in our cohort were hepatitis B infections, which was suitable for the
Chinese national condition. Although the subgroup analysis was taken to show that no matter what status of hepatitis B infection the result was consistent with the above, further subgroup analyses focused on the aetiological factors should be explored further.

In conclusion, the PLR is an independent risk factor of extrahepatic metastasis after radical hepatectomy for HCC patients. The high PLR indicates a higher rate of extrahepatic metastasis, which is also suitable for different BCLC stages, serum AFP level, and cirrhosis status. 

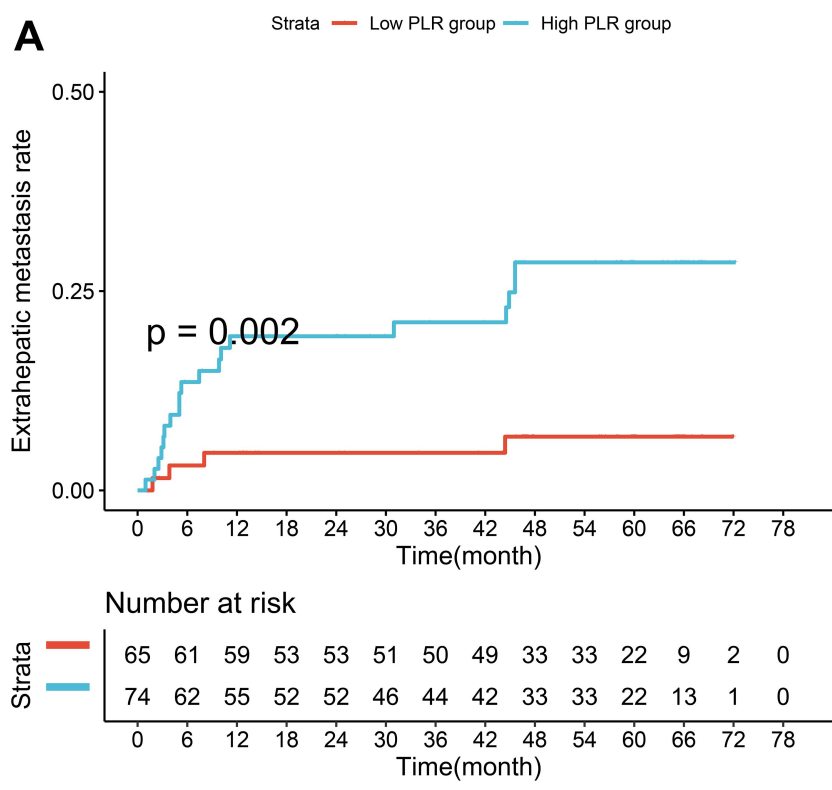

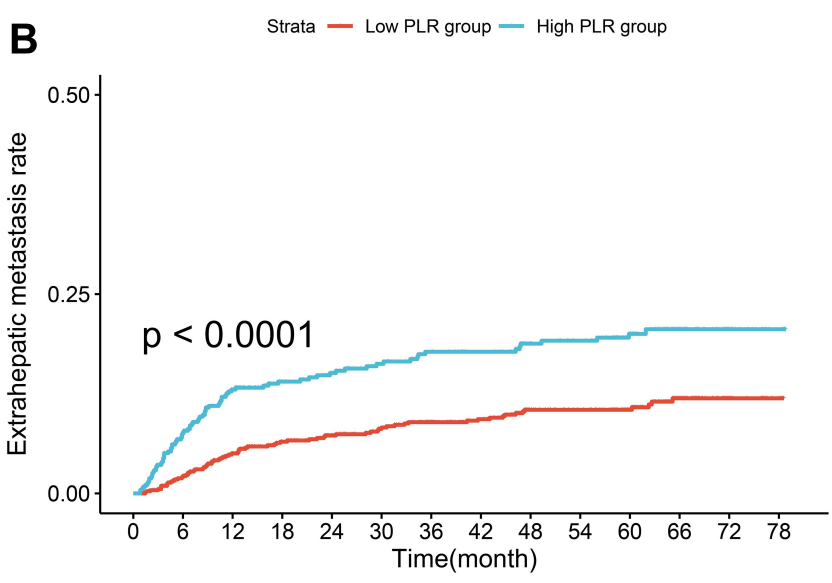

Number at risk

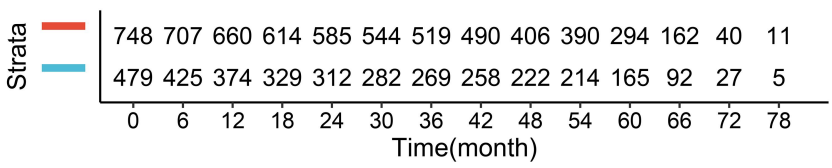

Figure 6 Subgroup analysis of metastasis rate between high and low PLR groups. (A) Metastasis rate in the HBsAg negative group; (B) metastasis rate in the HBsAg positive group.

Abbreviations: PLR, platelet-to-lymphocyte ratio; HBsAg, hepatitis B surface antigen.

\section{Abbreviations}

HCC, hepatocellular carcinoma; ROC, receiver operating characteristic; NLR, neutrophil-to-lymphocyte ratio; PNI, prognostic nutritional index; N $\gamma L R$, neutrophil times $\gamma$ glutamyl transpeptidase-to-lymphocyte ratio; PLR, platelet-to-lymphocyte ratio; LMR, lymphocyte-to-monocyte ratio; PNLR, platelet times neutrophil-to-lymphocyte ratio; AJCC, American Joint Committee on Cancer; BCLC, Barcelona Clinic Liver Cancer; AFP, alpha-fetoprotein; HBsAg, hepatitis B surface antigen; HBV-DNA, hepatitis B Virus deoxyribonucleic acid; RBC, red blood cell; HB, hemoglobin; TBIL, total bilirubin; PLT, platelet count; HR, hazard ratio; CI, confidence interval; SD, standard deviation; IQR, interquartile range.

\section{Ethical Statement}

All the patients were informed consent for their data to be used for research purposes. This study was approved by the institutional ethics committee of Mengchao Hepatobiliary Hospital of Fujian Medical University.

\section{Author Contributions}

All authors made substantial contributions to conception and design, acquisition of data, or analysis and interpretation of data; took part in drafting the article or revising it critically for important intellectual content; agreed to submit to the current journal; gave final approval of the version to be published; and agree to be accountable for all aspects of the work.

\section{Funding}

This study was supported by the Special Fund of Fujian Development and Reform Commission (31010308) and the Natural Science Foundation of Fujian Province (2018J01140).

\section{Disclosure}

The authors report no conflicts of interest in this work.

\section{References}

1. Torre LA, Bray F, Siegel RL, Ferlay J, Lortet-Tieulent J, Jemal A. Global cancer statistics, 2012. CA Cancer J Clin. 2015;65(2):87-108. doi: $10.3322 /$ caac. 21262

2. Sasaki A, Kai S, Endo Y, et al. Hepatitis B virus infection predicts extrahepatic metastasis after hepatic resection in patients with large hepatocellular carcinoma. Ann Surg Oncol. 2007;14(11):3181-3187. PMID: 17846843. doi:10.1245/s10434-007-9570-x

3. Tanaka K, Shimada H, Matsuo K, Takeda K, Nagano Y, Togo S. Clinical features of hepatocellular carcinoma developing extrahepatic recurrences after curative resection. World J Surg. 2008;32 (8):1738-1747. PMID: 18463920. doi:10.1007/s00268-008-96 13-x

4. Ochiai T, Ikoma H, Okamoto K, Kokuba Y, Sonoyama T, Otsuji E. Clinicopathologic features and risk factors for extrahepatic recurrences of hepatocellular carcinoma after curative resection. World J Surg. 2012;36(1):136-143. PMID: 22051887. doi:10.1007/s00268-011$1317-\mathrm{y}$ 
5. Byeon J, Cho EH, Kim SB, Choi DW. Extrahepatic recurrence of hepatocellular carcinoma after curative hepatic resection. Korean $J$ Hepatobiliary Pancreat Surg. 2012;16(3):93-97. PMID: 26388915; PMCID: PMC4575006. doi:10.14701/kjhbps.2012.16.3.93

6. Wang L, Ye G, Zhan C, et al. Clinical factors predictive of a better prognosis of pulmonary metastasectomy for hepatocellular carcinoma. Ann Thorac Surg. 2019;108(6):1685-1691. PMID: 31445050. doi:10.1016/j.athoracsur.2019.06.086

7. Llovet JM, Ricci S, Mazzaferro V, et al.; SHARP Investigators Study Group. Sorafenib in advanced hepatocellular carcinoma. $N$ Engl $J$ Med. 2008;359(4):378-390. PMID: 18650514. doi:10.1056/ NEJMoa0708857

8. Kudo M, Finn RS, Qin S, et al. Lenvatinib versus sorafenib in first-line treatment of patients with unresectable hepatocellular carcinoma: a randomised Phase 3 non-inferiority trial. Lancet. 2018;391(10126):1163-1173. PMID: 29433850. doi:10.1016/S0140-6736(18)30207-1

9. Wang Z, Wang J, Wang P. The prognostic value of prognostic nutritional index in hepatocellular carcinoma patients: a meta-analysis of observational studies. PLoS One. 2018;13(10):e0202987. PMID: 30312295; PMCID: PMC6185720. doi:10.1371/journal.pone.0202987

10. Fan W, Zhang Y, Wang Y, Yao X, Yang J, Li J. Neutrophil-to-lymphocyte and platelet-to-lymphocyte ratios as predictors of survival and metastasis for recurrent hepatocellular carcinoma after transarterial chemoembolization. PLoS One. 2015;10(3):e0119312. PMID: 25742141; PMCID: PMC4351002. doi:10.1371/journal.pone.0119312

11. Li M, Zhao Y, Liu X, Zhang S, Jiang Y, Yang Z. Early risk warning system for distant metastasis of hepatitis B virus-associated hepatocellular carcinoma with portal vein tumor thrombus. Oncol Lett. 2020;19(4):3249-3257. PMID: 32256820; PMCID: PMC7074481. doi:10.3892/ol.2020.11423

12. Ha Y, Mohamed Ali MA, Petersen MM, et al. Lymphocyte to monocyte ratio-based nomogram for predicting outcomes of hepatocellular carcinoma treated with sorafenib. Hepatol Int. 2020;14(5):776-787. PMID: 32740886. doi:10.1007/s12072-020-10076-4

13. Zhang T, Liu Z, Zhao X, Mao Z, Bai L. A novel prognostic score model based on combining systemic and hepatic inflammation markers in the prognosis of HBV-associated hepatocellular carcinoma patients. Artif Cells Nanomed Biotechnol. 2019;47(1):2246-2255. PMID: 31169437. doi:10.1080/21691401.2019.1573174

14. Li J, Liao Y, Suo L, et al. A novel prognostic index-neutrophil times $\gamma$-glutamyl transpeptidase to lymphocyte ratio $(\mathrm{N} \gamma \mathrm{LR})$ predicts outcome for patients with hepatocellular carcinoma. Sci Rep. 2017;7 (1):9229. PMID: 28835713; PMCID: PMC5569032. doi:10.1038/ s41598-017-09696-y

15. Jin $\mathrm{C}, \mathrm{Li} \mathrm{C}$, Peng $\mathrm{W}$, et al. Changes of platelet times neutrophil to lymphocyte ratio predict BCLC stage A hepatocellular carcinoma survival. Medicine. 2017;96(33):e7821. PMID: 28816981; PMCID: PMC5571718. doi:10.1097/MD.0000000000007821
16. Hanahan D, Weinberg RA. Hallmarks of cancer: the next generation. Cell. 2011;144(5):646-674. PMID: 21376230. doi:10.1016/j. cell.2011.02.013

17. Trinchieri G. Cancer and inflammation: an old intuition with rapidly evolving new concepts. Annu Rev Immunol. 2012;30:677-706. PMID: 22224761. doi:10.1146/annurev-immunol-020711-075008

18. Mouchli M, Reddy S, Gerrard M, Boardman L, Rubio M. Usefulness of neutrophil-to-lymphocyte ratio (NLR) as a prognostic predictor after treatment of hepatocellular carcinoma". Review article. Ann Hepatol. 2020;22:S1665-2681(20)30164-2. PMID: 32896610. doi:10.1016/j.aohep.2020.08.067

19. Yang Y, Nagano H, Ota H, et al. Patterns and clinicopathologic features of extrahepatic recurrence of hepatocellular carcinoma after curative resection. Surgery. 2007;141(2):196-202. PMID: 17263976. doi:10.1016/j.surg.2006.06.033

20. Galle PR, Forner A, Llovet JM, et al. EASL clinical practice guidelines: management of hepatocellular carcinoma. $J$ Hepatol. 2018;69:182-236.

21. Chun YS, Pawlik TM, Vauthey J-N. 8th edition of the AJCC cancer staging manual: pancreas and hepatobiliary cancers. Ann Surg Oncol. 2018;25:845-847. doi:10.1245/s10434-017-6025-x

22. Li J, Liu Y, Yan Z, et al. A nomogram predicting pulmonary metastasis of hepatocellular carcinoma following partial hepatectomy. $\mathrm{Br} \mathrm{J}$ Cancer. 2014;110(5):1110-1117. PMID: 24481404; PMCID: PMC3950869. doi:10.1038/bjc.2014.19

23. He C, Peng W, Liu X, Li C, Li X, Wen TF. Post-treatment alphafetoprotein response predicts prognosis of patients with hepatocellular carcinoma: a meta-analysis. Medicine. 2019;98(31):e16557. PMID: 31374020; PMCID: PMC6709300. doi:10.1097/ MD.0000000000016557

24. Bihari C, Rastogi A, Shasthry SM, et al. Platelets contribute to growth and metastasis in hepatocellular carcinoma. APMIS. 2016;124(9):776-786. PMID: 27457354. doi:10.1111/apm.12574

25. Chew V, Tow $\mathrm{C}$, Teo $\mathrm{M}$, et al. Inflammatory tumour microenvironment is associated with superior survival in hepatocellular carcinoma patients. $J$ Hepatol. 2010;52(3):370-379. PMID: 19720422. doi:10.1016/j.jhep.2009.07.013

26. Arroyo V, García-Martinez R, Salvatella X. Human serum albumin, systemic inflammation, and cirrhosis. J Hepatol. 2014;61(2):396407. PMID: 24751830. doi:10.1016/j.jhep.2014.04.012

27. Bernardi M, Moreau R, Angeli P, Schnabl B, Arroyo V. Mechanisms of decompensation and organ failure in cirrhosis: from peripheral arterial vasodilation to systemic inflammation hypothesis. $J$ Hepatol. 2015;63(5):1272-1284. PMID: 26192220. doi:10.1016/j. jhep.2015.07.004
Cancer Management and Research

\section{Publish your work in this journal}

Cancer Management and Research is an international, peer-reviewed open access journal focusing on cancer research and the optimal use of preventative and integrated treatment interventions to achieve improved outcomes, enhanced survival and quality of life for the cancer patient.
The manuscript management system is completely online and includes a very quick and fair peer-review system, which is all easy to use. Visit http://www.dovepress.com/testimonials.php to read real quotes from published authors. 